\title{
SPECTRUM SENSING PERFORMANCE OF COGNITIVE RADIO UNDER AWGN AND FADING CHANNEL CONDITIONS
}

\author{
K. Annapurna ${ }^{1}$, B. Seetha Ramanjaneyulu ${ }^{2}$ \\ ${ }^{1}$ Assistant Professor, ECE Department, Vignan's Univesrity, Andhra Pradesh, India \\ ${ }^{2}$ Professor, ECE Department, Vignan's Univesrity, Andhra Pradesh, India
}

\begin{abstract}
Accurate sensing of the spectrum occupancy is essential for successful implementation of cognitive radio networks. However, due to higher levels of noise and multipath propagation effects of the channel, the sensing information of cognitive radio may not be accurate all the times. In this context, Receiver Operating Characteristics (ROC) of the system gives a measure of this information. It is a curve of probability of true detection with respect to probability of false detection. This paper presents the ROC of cognitive radio system for AWGN, Rayleigh and Rician channels.
\end{abstract}

Keywords: Cognitive Radio, Spectrum Sensing, Primary User Detection, AWGN Channel, Rayleigh and Rician Channels

\section{INTRODUCTION}

Cognitive radio is an innovative solution to utilize the radio frequency spectrum efficiently. It allows the non-licensed users to use the spectrum opportunistically when the licensed user doesn't use it at that time [1-5]. Licensed users of the spectrum are termed as primary users (PUs) and unlicensed users as secondary users (SUs). Being the license holders of that spectrum, primary users can use their allotted channels at any time. But, for secondary user, before using any channel, it needs to sense the spectrum and find out the vacant channels of the spectrum available at that time. Then it takes a decision of availing one of those vacant channels. But the secondary user has to exercise enough care to vacate the channel when the primary user returns back to use that channel again. For this purpose, it has to keep sensing the channel continuously to find out whether the primary user has returned or not. Hence, spectrum sensing is one of the key mechanisms to be carried out properly for efficient functioning of cognitive radio. The accuracy of this sensing decides whether the vacant channels are correctly identified or the presence of primary users is properly detected. This accuracy depends on several factors including the type of channels being sensed. As wireless communications suffer from problems like multipath propagation and fading in many situations, assessing the cognitive radio receiver's spectrum sensing performance in these environments in comparison with a channel of Additive White Gaussian Noise (AWGN) is helpful to predict the detection accuracies for various levels of interference. This performance assessment is carried out in this work. Section-2 discusses the concept of spectrum sensing along with fundamental spectrum sensing types. Section-3 covers the channel conditions of wireless communication and section-4 describes the concept of ROC used for detecting the presence of primary user of the spectrum. Simulation results of detecting performance for various channel conditions are presented in section-5 and section- 6 concludes the work.

\section{SPECTRUM SENSING AND DETECTION OF PRIMARY USER}

The process of detecting the used and unused portions of the spectrum with respect to time and location is called spectrum sensing in cognitive radio systems. It should be carried out continuously by cognitive radios, for two reasons. The first reason is to know about the opportunities that arise from time-to-time and the other reason is to vacate the occupied channel whenever the primary user of the occupied channel returns back to use it.

Since the PUs have the authority of using the spectrum directly without any time restrictions, SUs need to observe the activities of PUs continuously to get the information of spectrum holes that become available. 'Spectrum holes' are those frequency bands which are identified as not used by the PUs. There are two types of spectrum holes namely temporal and spatial, based on availability of spectrum with respect to time and area respectively [6].

The presence or absence of PUs can be found by the cognitive radio by detecting the PU transmitter or PU receiver or PU signals. Among these methods, detecting the PU signals is simple and straight forward method compared to detecting PU transmitter and PU receiver [10]. Hidden terminal effect is one of the main problems associated with detecting the PU transmitter or receiver. Hence detecting the PU signal can be appropriate choice.

A point that needs to be mentioned here is that the secondary device cannot distinguish the PU signal from its co-SU signals. It treats both of them as the presence of signal in that channel at that time, which conveys the 
meaning that the channel is occupied. If this signal that represents the channel occupancy is considered as $x(t)$, then the signal detected by the SU at any instant of time can be considered as $\mathrm{y}(\mathrm{t})$, which it can be represented as [7],

$$
y(t)=\begin{aligned}
& n(t) \ldots \ldots \ldots \ldots \ldots H_{0} \\
& x(t)+n(t) \ldots \ldots \ldots H_{1}
\end{aligned}
$$

where $n(t)$ is noise, and $\mathrm{H}_{0}$ and $\mathrm{H}_{1}$ are the hypotheses of absence or presence of PU signals or other SU signals respectively. The objective of spectrum sensing is to decide on $\mathrm{H}_{0}$ or $\mathrm{H}_{1}$ based on received signal $\mathrm{y}(\mathrm{t})$.

There are multiple ways of sensing the spectrum, of which energy detection, matched filter (coherent) detection and cyclo-stationary feature detection are most popular techniques. Energy detection [8] method is simple and efficient. The main limitation of this technique is its performance is good only when SNR is high. The average energy of observed signal is considered here which can be represented as,

$$
y=\frac{1}{N} \sum_{t=1}^{N} y(t)^{2}
$$

where, $\mathrm{N}=$ number of samples.

This average signal energy is compared with a pre defined threshold value $(\gamma)$ to decide the presence of PU. If this energy is greater than the threshold value $(y>\gamma)$, PU is considered to be present; otherwise it is considered to be absent. The selection of threshold plays an important role. If the threshold value is set to too low, then elevated noise energy levels also can be represented as the presence of PU, though it is not there. If this value is set too high, then there is a problem of not identifying the PU signal even it is actually present.

This problem can be overcome, if the SU has prior knowledge of PU signals. In that case, matched filter detection or coherent detection [8] can be used. In this method, the received signal is convolved with the filter whose impulse response is the time shifted mirror image of reference signal. It needs very less sensing time, but requires the prior knowledge of PU signals, which may not be possible in many real time cases. The convolution of received signal and impulse response of the filter can be expressed as

$$
z(n)=\sum_{k=-\infty}^{\infty} h(n-k) \cdot y(k)
$$

$Z(n)$ will be maximum if the PU signal is present and minimum in its absence.

Cyclo-stationary feature detection method [9-10] is another promising technique that checks for the periodicity in the detected signal. As periodicity is a distinguishing characteristic of signals but not of the noise, it gives good results even for low SNR values. But the complexity of the circuit is high for this method. It can be realized by analyzing the cyclic auto correlation function of received signal $\mathrm{x}(\mathrm{t})$, expressed as,

$$
R_{y}^{(\beta)}(\tau)=E\left(y(t) * y(t-\tau) e^{-j 2 \pi \beta t}\right.
$$

where $\mathrm{E}($.$) is expectation operator used to find statistical$ average and $\beta$ is cyclic frequency. ${ }^{R_{y}^{(\beta)}}(\tau)$ will be maximum for the presence of signal and minimum for its absence.

\section{CHANNEL MODELS}

In addition to the method used to detect the signal, channel characteristics also play an important role in deciding the spectrum sensing performance. In mobile communication environment, multipath fading $[11,12]$ is a common problem. This problem occurs due to the multiple copies of the signal that reach the destination by travelling through different paths and so having different phase shifts and attenuation levels. The reasons for multipath propagation are atmospheric ducting, Ionospheric reflection and refraction, reflection from water bodies, reflection from terrestrial objects etc. Multipath fading can be constructive or destructive based on phase shift. 'Rayleigh' and 'Rician' fadings are the two types of channel conditions that describe these multipath fading channels. ' $\mathrm{k}$ ' factor is used to categorize the given channel. It is defines as,

$$
\text { ' } k \text { ' factor }=\frac{\text { Power in the domnant path }}{\text { Power in the scattered paths }} .
$$

If ' $\mathrm{k}$ ' factor is ' 0 ', it means there is no dominant path and that channel is referred to as Rayleigh fading channel. If there is a dominant path or line of sight (LOS) path, then $\mathrm{k}>0$ and that channel is referred to as Rician fading channel.

So, Rayleigh fading channel is that in which there are multiple indirect paths between transmitter and receiver and no dominant path such as Line Of Sight (LOS) path. This channel condition can be considered as the worst type, and is an example of outdoor communication. Rician fading channel is that in which there are multiple indirect paths between transmitter and receiver along with a dominant path such as LOS path. This condition is applicable to indoor environments.

Additive White Gaussian Noise (AWGN) channel is also considered here for discussion. In AWGN channel, only one copy of the signal is present at the destination, and so the value of ' $k$ ' can be considered as 'infinity'. Thermal noise and attenuation thereof is the only problem in this kind of channel. This type of channel is present in space communications or coaxial cable like communications. It is discussed here for comparison purpose only to compare the 'Rayleigh' and 'Rician' channels with AWGN channel. Rayleigh fading channel and AWGN channel models can be 
considered as special cases of Rician fading channel model, with ' $k$ ' factor of ' 0 ' and ' $\infty$ ' respectively. Spectrum sensing performance of cognitive radio under these three conditions is carried out in this work.

\section{RECEIVER OPERATING CHARACTERISTICS}

Receiver Operating Characteristics curves (ROC curves) are very popular for binary classification problems. They can be used here also, to know the sensing performance of cognitive radios that use energy detection method for spectrum sensing. These curves are drawn for sensitivity versus complementary of specificity $[13,14]$.

Sensitivity is a measure of detecting a signal when the signal is present. It can be represented as,

$$
\text { sensitivity }=\frac{\text { True positive }}{\text { True positive }+ \text { False negative }} \text {. }
$$

Specificity is a measure of not detecting it, when the signal is absent. It can be represented as,

$$
\text { specificity }=\frac{\text { True negative }}{\text { True negative }+ \text { False positive }} \text {. }
$$

It can be expected that both sensitivity and specificity should be high for a cognitive radio's spectrum sensing unit, or for that matter for any binary classifying system. It can be explained with the help of binary symmetric channel model shown in Fig-1. The possible inputs and outputs of it are either a ' 0 ' or a ' 1 '. Here let the ' 0 ' and ' 1 ' on the input side indicate absence and presence of PU, and ' 0 ' and ' 1 ' on the output side indicate the detection of absence and presence of PU respectively. The various possibilities in this model are:

- $\quad$ True Positive $(\mathrm{TP})=$ Detecting the presence of PU when it is present actually

- $\quad$ True Negative $(\mathrm{TN})=$ Detecting the absence of PU when it is not present actually

- $\quad$ False Positive (FP) = Detecting the presence of PU when it is not present actually

- $\quad$ False Negative $(F N)=$ Detecting the absence of PU when it is present actually

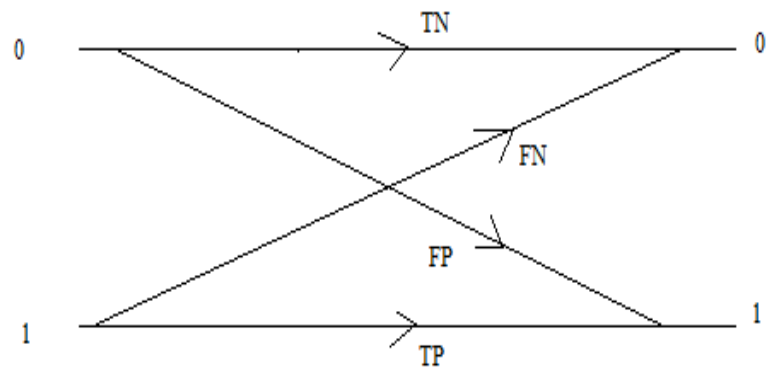

Fig-1: Binary Symmetric Channel model indicates more false alarms, which means detecting the presence of signal even though there is no signal, which results in missing of opportunities to secondary users. So specificity should also be high in order to not to miss the opportunities.

An ROC plot depicts the tradeoff between benefits (no interference) and costs (missing opportunities) [13] in cognitive radio system. Ideal value of area under ROC is ' 1 ', where the characteristics will be very close to sensitivity axis. The area under ROC is a measure of correct classification [15], that is the indication of amount correctly classifying signal presence and absence. ROC for three types of channels namely AWGN channel, Rayleigh channel and Rician channel are obtained.

\section{SIMULATION RESULTS}

Spectrum sensing for three different channel types is simulated using MATLAB. Sensitivity and specificity are calculated and ROCs are plotted. For AWGN, different ROCs are obtained for different SNR values like 10, 15 and $20 \mathrm{~dB}$. It is shown in Fig-2. It is observed that higher SNR values offer better detection performance.

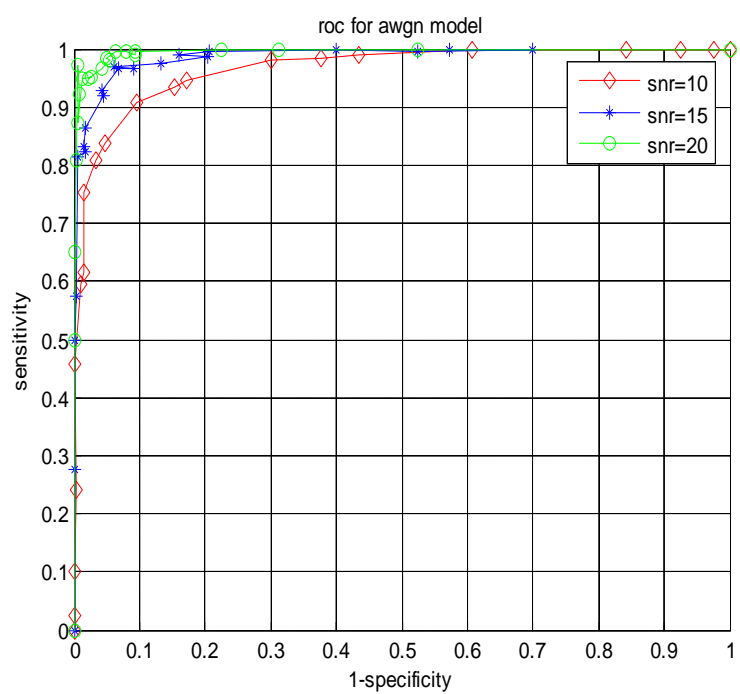

Fig-2: ROC characteristics for AWGN channel model

In the second case the transmitted signal is passed through Rayleigh fading channel, for which ROCs are plotted for three different values of Doppler shifts as shown in Fig-3. Doppler shift is the change in frequency of a wave (or other periodic event) for an observer moving relative to its source. It is observed that less Doppler shift results in better performance.

High sensitivity indicates less interference to PUs. A sensitivity of 1 indicates no interference to PUs. So sensitivity should be as high as possible. Less specificity 


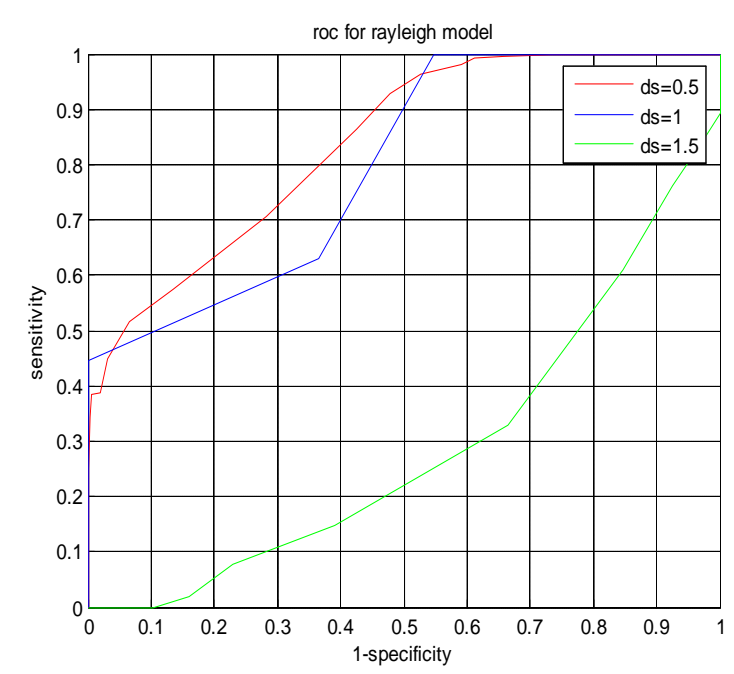

Fig-3: ROC characteristics for Rayleigh channel model

In the third case the transmitted signal is passed through Rician fading channel, for which ROCs are plotted for three different values of $\mathrm{k}$-factors as shown in Fig-4. It is observed that a high $\mathrm{k}$-factor gives better performance. It can be observed that as ' $\mathrm{k}$ ' value increases, its ROC curve resembles close to that of AWGN.

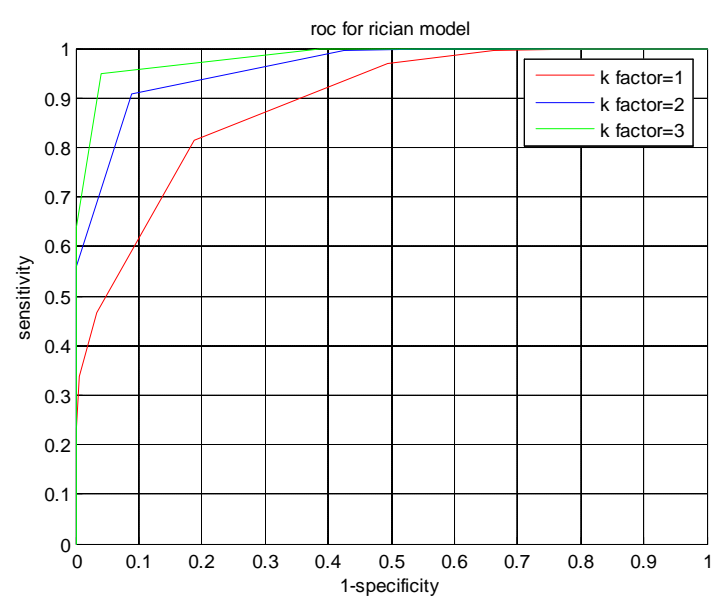

Fig-4: ROC characteristics for Rayleigh channel model

\section{CONCLUSION}

Spectrum sensing performance of cognitive radios is evaluated for various channel conditions like AWGN, Rayleigh and Rician channels. Receiver Operating Characteristics (ROC) of the cognitive radios operating under these channel conditions are obtained and plotted for various SNR values, doppler shifts and ' $k$ ' factor values. It is observed that the effect of Rician channel is less when compared to Rayleigh channel. As expected, performance of the radio in AWGN channel is better than both Rician and Rayleigh channels. This evaluation provides an insight into the probable accuracies of spectrum detection in these channel conditions.

\section{REFERENCES}

[1] J Mitola, "Software radios: survey, critical evaluation and future directions", IEEE Aerospace and electronic system magazinevol.8no.4, pp. 25-36, Apr 1993.

[2] J Mitola, GQ Maguire, "Cognitive radio: making software radios more personal", IEEE Personal Communications, vol.6, no.4, pp.13-18, 1999.

[3] S Haykin, "Cognitive radio: Brain-empowered wireless communication", IEEE Selected Areas in Communications", vol.23,no.2, pp.201-220, 2005

[4] FK Jondral, "Software-defined radio-basics and evolution to cognitive radio" EURASIP Journal on wireless communications and networking, pp.275283, 2005.

[5] Ian.F Akyildiz, Won-YeolLee, Mehmet C.Vuran, Shantidu Mohanty, "Next generation/dynamic spectrum access/ cognitive radio wireless networks: A survey. Comput Netw”, vol.50, no. 13, sept 2006.

[6] J Ma, GY Li, BH Juang, "Signal processing in cognitive radio" Proc IEEE, vol.97,no.5, pp. 805823, 2009.

[7] A.Ghasemi, E.S, Sousa, "Impact of user collaboration on the performance of opportunistic spectrum schemes", in proc IEEE vehicular Technology Conference, pp.1-6, 2006.

[8] Anant Sahai, Niels.Hoven, Rahul Tandra, "Some fundamental limits on cognitive radio", in proc Allerton con Communication Control Computer, 2014.

[9] M.Ghozzi, F.Marx, M.Dohler, J.Palicot, "cyclo stationary based test for detection of vacant frequency bands", in proc2nd int conf on cognitive radio oriented wireless Networks and Communications, pp.1-5, 2006.

[10] PD Suttan, K.E. Nolan, LE Doyle, cyclostationary signature in practical cognitive radio applications", IEEE Journal on Selected Areas in Communications, vol.26, no.1, Jan 2008.

[11] William Stallings, “ wireless communications and networks", pearson publications, $2^{\text {nd }}$ edition.

[12] Nuzhat Tasneem Awon, Md. Mizanur Rahman, Md. Ashraful Islam, A.Z.M. Touhidul Islam, "Effect of AWGN \& Fading (Raleigh \& Rician) channels on BER performance of a WiMAX communication System", (IJCSIS) International Journal of Computer Science and Information Security,Vol.10, No.8, pp:11-17, Aug 12.

[13] Tom Fawcett, "An introduction to ROC analysis", pattern recognition letters, pp: 861-874, 2006.

[14] Charles E Metz, "Basic principles of ROC Analysis", seminars in nuclear medicine, Vol. 8, No.4, Oct 1978.

[15] James A. Hanley, Barbarac J. Mc Neil, "The meaning and use of the Area under a Receiver Operating Characteristic (ROC) curve, copyright 1982 by the Radiological Society of North America Incorporated, pp.29-36, Apr 1982. 


\section{BIOGRAPHIES}

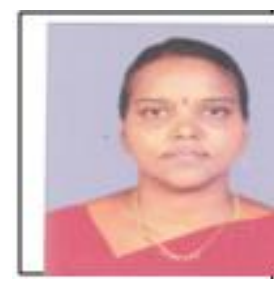

Mrs. K Annapurna received her

B.Tech in ECE from Acharya Nagarjuna University in 2001, M.Tech in DSCE from JNTUH in 2011 and currently pursuing her Ph.D in the area of cognitive radio networks in Vignan's University. Her research interests are cognitive radio networks and image processing.

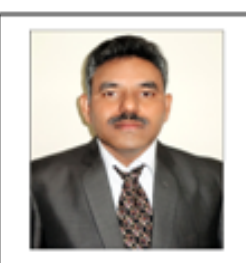

Dr. B Seetha Ramanjaneyulu received his B.E. in ECE from Andhra University in 1991 and M.E., in Electronics Engineering from Bangalore University in 1993, and Ph.D., from NIT Calicut in 2007. He worked as Senior Design Engineer and Scientist in organizations like CDAC and National Institute of Electronics and IT, before joining the Vignan's University in 2008. His research interests include Computer Networks, Sensor Networks, Wireless Communications, High-Performance Computing and Network Security. 